Review paper

\title{
An optimized calibration method for surface measurements with MOSFETs in shaped-beam radiosurgery
}

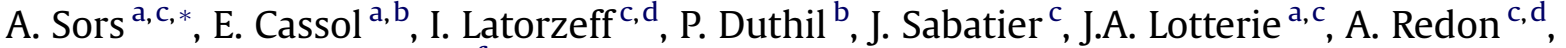 \\ I. Berry ${ }^{a, c}, X$. Franceries ${ }^{a, e, f}$
}

a Inserm, Imagerie cérébrale et handicaps neurologiques, UMR 825, F-31059 Toulouse, France

${ }^{\mathrm{b}}$ Unité de Radiophysique et de Radioprotection, CHU Toulouse, France

${ }^{\mathrm{c} C e n t r e ~ d e ~ R a d i o c h i r u r g i e ~ S t e ́ r e ́ o t a x i q u e, ~ B i o p h y s i q u e ~ M e ́ d i c a l e ~-~ C H U ~ R a n g u e i l, ~ a v . ~ J . ~ P o u l h e ̀ s ~-~ T S A ~ 50032, ~} 31059$ Toulouse Cedex 9, France

${ }^{\mathrm{d}}$ Groupe Oncorad Garonne, France

e Université de Toulouse, UPS, INPT, LAPLACE Laboratoire Plasma et Conversion d'Energie, 118 route de Narbonne, F-31062 Toulouse Cedex 9 , France

${ }^{\mathrm{f}}$ Universitéde Toulouse, UPS, Imagerie cérébrale et handicaps neurologiques, UMR 825, F-31059 Toulouse, France

\section{A R T I C L E I N F O}

\section{Article history:}

Received 6 October 2012

Received in revised form

11 March 2013

Accepted 14 March 2013

Available online 23 April 2013

\section{Keywords:}

In-vivo dosimetry

MOSFET

Radiosurgery

Small field dosimetry

\begin{abstract}
A B S T R A C T
Nowadays MOSFET dosimeters are widely used for dose verification in radiotherapy procedures. Although their sensitive area satisfies size requirements for small field dosimetry, their use in radiosurgery has rarely been reported. The aim of this study is to propose and optimize a calibration method to perform surface measurements in $6 \mathrm{MV}$ shaped-beam radiosurgery for field sizes down to $18 \times 18 \mathrm{~mm}^{2}$. The effect of different parameters such as recovery time between 2 readings, batch uniformity and build-up cap attenuation was studied. Batch uniformity was found to be within $2 \%$ and isocenter dose attenuation due to the build-up cap over the MOSFET was near $2 \%$ irrespective of field size. Two sets of sensitivity coefficients (SC) were determined for TN-502RD MOSFET dosimeters using experimental and calculated calibration; the latter being developed using an inverse square law model. Validation measurements were performed on a realistic head phantom in irregular fields. MOSFET dose values obtained by applying either measured or calculated SC were compared. For calibration, optimal results were obtained for an inter-measurement time lapse of $5 \mathrm{~min}$. We also found that fitting the SC values with the inverse square law reduced the number of measurements required for calibration. The study demonstrated that combining inverse square law and Sterling-Worthley formula resulted in an underestimation of up to $4 \%$ of the dose measured by MOSFETs for complex beam geometries. With the inverse square law, it is possible to reduce the number of measurements required for calibration for multiple field-SSD combinations. Our results suggested that MOSFETs are suitable sensors for dosimetry when used at the surface in shaped-beam radiosurgery down to $18 \times 18 \mathrm{~mm}^{2}$.
\end{abstract}

(C) 2013 Associazione Italiana di Fisica Medica. Published by Elsevier Ltd. All rights reserved.

\section{Introduction}

Nowadays MOSFET (Metal-Oxide Semiconductor Field-Effect Transistor) dosimeters are widely used for dose verification in radiotherapy procedures. But in narrow beams, flatness of the crossbeam profile is only achieved over a small fraction of the fullwidth half maximum [1]: most of the field is penumbra, and the dose received by the detector may fall off significantly from the center to the periphery [2]. The ideal dosimeter should present

\footnotetext{
* Corresponding author. Centre de Radiochirurgie Stéréotaxique, Biophysique Médicale - CHU Rangueil, av. J. Poulhès - TSA 50032, 31059 Toulouse Cedex 9, France. Tel.: +33 (0)561341252; fax: +33 (0)561322754.

E-mail address: sors.aurelie@hotmail.fr (A. Sors).
}

the following characteristics: tissue equivalent, energy/dose rate independent, minimal field perturbation, very high spatial resolution and a small sensitive volume to minimize the volume averaging effect and to avoid measurement artifacts [3]. Small field dosimetry still poses a problem for measurements of Total Scatter Factor $\left(S_{\mathrm{cp}}\right)$, TMR and OAR parameters especially with the use of traditional ionization chambers for field diameters less than about $17 \mathrm{~mm}$ [4]. For diameters down to $8 \mathrm{~mm}$ [5], better results seem to be obtained using diodes, TLDs or films to determine the physical parameters mentioned above. Moreover, in clinical conditions, the detector has to be convenient, well suited to small field requirements and the irregular skin surface of the head. In consequence, diodes and films are not suitable. The use of TLD could be an alternative but several studies have reported their fragility and the requirement for lengthy calibration and tedious readout 
procedure [6]. The sensitive area of a MOSFET is so small that it can be considered as a point measurement. Therefore, MOSFETs could be suitable for in-vivo assessment of radiosurgery microbeams $[4,6,7]$. Additional studies have reported excellent linearity, dosimetric accuracy in the build-up region and directional independence $[8,9]$. Besides, MOSFETs have no dose rate dependence $[8,10,11]$ and can be read in real time.

Several MOSFET calibration methods are described in the literature with numerous correction/conversion factors applied to the measurements given by the detector. MOSFETs are generally calibrated under full build-up conditions, i.e. inserted into the phantom for a $10 \times 10 \mathrm{~cm}^{2}$ field size, placed at $D_{\max }$ depth [11] or deeper [12]. The disadvantage of this method is that calibration is performed under conditions which differ from clinical use when dosimeters are located at the surface to perform in-vivo measurements. An alternative method is to position the MOSFET, equipped with build-up cap, at the phantom surface. MOSFETs located at the surface have to be individually calibrated when they are equipped with build-up cap, whereas in-depth, up to five MOSFETs can be calibrated at once [13]. Most of the time, MOSFET dose is defined either from simultaneous ionization chamber measurements or from previously defined LINAC output [14], corrected for the daily output factor.

Calibrating MOSFETs for use in stereotactic radiosurgery surface measurements requires special considerations. Indeed, using a unique calibration coefficient under a $10 \times 10 \mathrm{~cm}^{2}$ reference field size constitutes a limitation to characterize precisely the detector in small field conditions. Further correction factors must be added to the measurement. Therefore, additional measurements have to be performed for typical field sizes encountered in stereotactic radiosurgery. However, this can considerably lengthen the overall calibration procedure and increases customer cost, since the end of the MOSFET's useful life of $20,000 \mathrm{mV}$ is reached more quickly. Therefore, there is a need to develop a calibration method satisfying stereotactic radiosurgery requirements, calibration time minimization and MOSFET lifespan preservation for the use of detectors located at the surface. There is no published study describing how to perform calibration when MOSFET is used as in-vivo dosimeters located at the surface of the calibration phantom in small fields. Invivo dosimetry for intracranial radiosurgery treatments presents an urgent challenge because they are usually delivered in a single fraction with small fields. So, to evaluate the use of MOSFETs (TN502RD type) in shaped-beam radiosurgery, the aim of this work is to propose an optimized calibration method for field sizes down to $18 \times 18 \mathrm{~mm}^{2}$

\section{Material and methods}

\section{Dosimetry system and detectors studied}

The study was performed on a Novalis (Varian) accelerator equipped with an $\mathrm{m} 3$ micro-MLC (BrainLab ${ }^{\circledR}$ ) delivering a $6 \mathrm{MV}$ photon beam. The treatment planning system (TPS), used was BrainScan version 5.3.1 $\left(\right.$ BrainLab $\left.^{\circledR}\right)$, using a pencil-beam algorithm for dose computation. The mobileMOSFET dose verification System (TN-RD-70W, Best Medical Canada, Ottawa, Canada) was used as a dose measurement and readout device. It is composed of a small reader volume which communicates wirelessly to a computer using a Bluetooth transceiver module. The MOSFET dosimeters used in this study are p-channel and of the dual-MOSFET type (TN-502RD type, Best Medical Canada, Ottawa, Canada). One to five MOSFET detectors can be connected to the reader at once. The MOSFETs studied had the following dimensions: $1.3 \mathrm{~mm}$ (thickness) $\times 2.5 \mathrm{~mm}$ (width) $\times 8 \mathrm{~mm}$ (length). The dimensions of the sensitive detector area were $0.2 \mathrm{~mm} \times 0.2 \mathrm{~mm}$. The MOSFET threshold voltage shift $\left|\Delta V_{\text {th }}\right|$, due to irradiation, is proportional to dose deposition [15]. The
MOSFET detector can be used up to $20,000 \mathrm{mV}$ in threshold voltage change with an acceptable linear response, reported to be within $4 \%$ at ${ }^{60}$ Co energy [16]. The associated maximum dose measured depends on the reader bias sensitivities selected. Thus, the associated maximum dose measured for standard sensitivity $\left(1 \mathrm{mV} \mathrm{cGy}{ }^{-1}\right)$ is approximately $200 \mathrm{~Gy}$ and $66 \mathrm{~Gy}$ for high sensitivity $\left(3 \mathrm{mV} \mathrm{cGy}^{-1}\right)$.

The radiation measurement cycle consists of pre-irradiation reading of dosimeters (initialization), followed by dose reading immediately after irradiation delivery. MOSFETs were readout $30 \mathrm{~s}$ after each irradiation in order to obtain a stable readout without fading effect. For calibration studies, the inter-measurement cycle intervals tested were 3 and $5 \mathrm{~min}$ in order to find the best compromise between fast measurement sequences and optimal reproducibility in sensitivity coefficient determination. For preclinical head phantom measurements, the inter-measurement cycle interval was set to $3 \mathrm{~min}$, corresponding to the minimum required time for LINAC and MOSFET repositioning. A maximum inter-measurement cycle interval of 5 min was chosen, which was in agreement with the manufacturer's recommendations of 25 min [17]. For pre-clinical measurements performed on head phantom, the inter-measurement cycle interval was thus set to 3 min. MOSFET dosimeters were used in standard bias mode (Nominal sensitivity of $1 \mathrm{mV} \mathrm{cGy}{ }^{-1}$ ) to extend the lifespan of the device.

\section{Calibration}

All dose measurements performed during calibration were done using MOSFETs of the same batch. Measurements were made on the same day after measuring the accelerator output. The system was warmed up for approximately $60 \mathrm{~min}$ before use, as recommended [8]. Detectors were calibrated at the surface of an XWU-IMRT Phantom (Best Medical Canada, Ottawa, Canada; $\left.\rho=1.18 \mathrm{~g} \mathrm{~cm}^{-3}, 20 \times 20 \times 20 \mathrm{~cm}^{3}\right)$. For all measurements, the sensor was oriented with the dome upwards toward the radiation source.

The MOSFET was covered by an RW3 hemispherical build-up cap $\left(\rho=1.04 \mathrm{~g} \mathrm{~cm}^{-3}\right.$ and diameter $\left.=13 \mathrm{~mm}\right)$ in a close-fitted groove to avoid air gaps. From the geometrical dimensions of the cap and detector, the total build-up water-equivalent thickness above the dosimeter's active area was estimated to be $7.1 \mathrm{~mm}$ (inherent build-up thickness was determined to be $0.8 \mathrm{~mm}$ at $6 \mathrm{MV}$ ). PDD curves were measured under reference conditions with a PTW 31014 PinPoint ${ }^{\circledR}$ type ionization chamber (volume $0.015 \mathrm{~cm}^{3}$ ). For each field size ranging from $98 \times 98$ down to $18 \times 18 \mathrm{~mm}^{2}$, the area under the PDD curve was computed from the surface to the depth of maximum dose $\left(A_{\max }\right)_{\text {field }}$ size using the trapezoid method from MATLAB software computations (MATLAB, MathWorks $\left.{ }^{\odot}\right)$. By using equally spaced node formalism, subintervals were defined in step sizes of $0.5 \mathrm{~mm}$. With the same numerical integration method, the area under the PDD curve was computed from the surface to a depth of water-equivalent thickness induced by the corresponding build-up caps i.e. $7.1 \mathrm{~mm}$, $\left(A_{7.1}\right)_{\text {field }}$ size. To estimate the charge particle equilibrium achievement due to the build-up cap, ratio of $\left(A_{7.1}\right)_{\text {field size }} /\left(A_{\text {max }}\right)_{\text {field size }}$ was computed. Dose depositions (\%) were extracted from PDD profiles from $98 \times 98$ to $18 \times 18 \mathrm{~mm}^{2}$ of each field size at depth of $7.1 \mathrm{~mm}$ and compared. Dose attenuation due to the MOSFET build-up cap was measured at 1 Gy for each field size by a PTW 31014 PinPoint ${ }^{\circledR}$ type ionization chamber (volume $0.015 \mathrm{~cm}^{3}$ ) placed at the depth of $D_{\max }$.

Measured Sensitivity Coefficients $\left(\mathrm{SC}_{\mathrm{m}}\right)$ in $\mathrm{mV} \mathrm{cGy}{ }^{-1}$ were acquired experimentally at different SSDs and for different field sizes from the average of 3 measurements. The MOSFET is at the phantom surface for all these measurements. By changing SSD values 
(SSD $=87,90,92,95,97,98.5 \mathrm{~cm}$ ), while maintaining isocentric conditions, $100 \mathrm{~cm}$ from the source, the isocenter was located at different depths in the center of the cross-section of the phantom. MUs were computed from TPS to deliver a dose of $2.5 \mathrm{~Gy}$ for each SSD-field size combination.

To cover methodological considerations related to measurement accuracy, several measurements were performed:

- Effect of inter-measurement cycle interval of 3 and 5 min on $\left(\mathrm{SC}_{\mathrm{m}}\right)$ variability was studied.

- Batch uniformity was estimated at 100 monitor units (MU) of radiation exposure using the standard deviation calculation for 3 -average readings of the dosimeter positioned at the surface and at $98.5 \mathrm{~cm}$ SSD. Measurements were obtained from 5 MOSFETs of the same batch at different points in their lifetime. The lifespan of $20,000 \mathrm{mV}$ was divided by 11 in order to obtain 10 different ages intervals. For the $k$ th MOSFET of the same batch $(k=1-5)$, measurements were taken from the $k$ th and the 11- $k$ th ages intervals (Table 1 ) to randomly cover the useful lifespan of detectors.

A matrix $M_{\mathrm{m}}$ was generated from the $\mathrm{SC}_{\mathrm{m}}$ values using a $2 \mathrm{D}$ bilinear interpolation function computed with Matlab Software. The matrix contains 101,598 nodes linking SSD (from 87 to $98.5 \mathrm{~cm}$ ) and square field sizes (from $18 \times 18$ to $98 \times 98 \mathrm{~mm}^{2}$ ). The interpolation step was $0.1 \mathrm{~mm}$ for field size and $1 \mathrm{~mm}$ for SSD. From measurements performed at SSD $=98.5 \mathrm{~cm}$, the inverse square law prediction was used to find the expected voltage shift values $\left(V_{\mathrm{th}}\right)$ using the inverse square correction factor. The elements for matrix $M_{\mathrm{c}}$ were calculated as follows. For a given field size:

$\left(\Delta V_{\mathrm{th}}\right)_{\mathrm{x}}=\left(\mathrm{SSD}_{98.5}\right)^{2} /\left(\mathrm{SSD}_{\mathrm{x}}\right)^{2} \times\left(\Delta V_{\mathrm{th}}\right)_{98.5}$

where: $\mathrm{SSD}_{98.5}$ corresponds to $\mathrm{SSD}=98.5 \mathrm{~cm}, \mathrm{SSD}_{\mathrm{x}}$, corresponds to $\mathrm{SSD}=\mathrm{x} \mathrm{cm},\left(V_{\mathrm{th}}\right)_{98.5}$ is the voltage shift obtained at $\mathrm{SSD}=98.5 \mathrm{~cm}$, $\left(V_{\text {th }}\right)_{\mathrm{x}}$ is the voltage shift obtained at $\mathrm{SSD}=\mathrm{x} \mathrm{cm}$.

The values of $\left(V_{\mathrm{th}}\right)_{\mathrm{x}}$ were divided by the prescribed dose to compute the corresponding calculated sensitivity coefficients $\left(\mathrm{SC}_{\mathrm{c}}\right)$ in $\mathrm{mV}_{\mathrm{cGy}}{ }^{-1}$. If the inverse square correction factor can be validated, the $6 \mathrm{SC}_{\mathrm{m}}$ values measured at different field sizes are adequate for calibration purposes, since the effect of varying SSD can be determined from inverse square correction factor. Therefore, the time and number of measurement can be reduced.

For both short and long inter-measurement cycle intervals, total calibration time obtained using either complete or optimized methods was estimated. Comparison between $\mathrm{SC}_{\mathrm{c}}$ and $\mathrm{SC}_{\mathrm{m}}$ was performed using the Pearson correlation coefficient $\left(R^{2}\right)$.

\section{Clinical validation measurements}

Clinical validation measurements were performed on a head phantom containing solid water, bone and vascular inserts in the right hemispherical part of the brain. As shown in Fig. 1, MOSFETs covered with build-up caps, were centered on the beam axis for all gantry rotations studied. All measurements were performed with MOSFETs coming from the same calibrated batch. The effect of beam geometry on the MOSFET response was studied as described

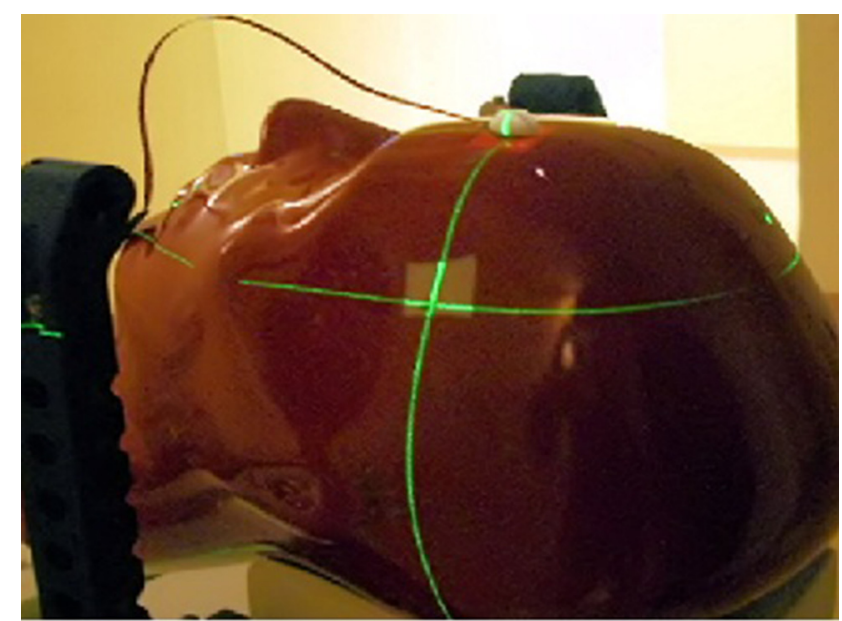

Figure 1. MOSFET positioning on the beam axis after verification of head phantom positioning using the orthovoltage X-rays generated by the ExacTrac System $\left(\right.$ BrainLab $\left.^{\circledR}\right)$.

below. For both regular and irregular field shapes, one measurement was performed for each field size-gantry angle combination studied. Between each geometrical irradiation condition, a waiting time of 3 min was applied. Only $\mathrm{SC}_{\mathrm{m}}$ determined using a waiting time of 5 min were used for clinical measurements on the head phantom. For in-vivo dosimetry, it was reported that it is possible to use entrance dose measurements, if it they are converted to the corresponding target dose using patient and treatment setup information $[13,18-20]$.

Then $\mathrm{SC}_{\mathrm{m}}$ and $\mathrm{SC}_{\mathrm{c}}$ were applied to MOSFETs measurements performed on a head phantom to simulate clinical conditions of surface measurements in regular (3 symmetric, 1 asymmetric) and in irregular field shapes.

Measurements were performed in static conditions, without stressing the m3 MLC (without opening and closing leaves fully continuously for several cycles). For clinical validation measurements performed also in static conditions, leaf positioning accuracy and repeatability of the $\mathrm{m} 3$ micro-MLC is well within the tolerance limit of $0.1 \mathrm{~cm}$.

\section{Regular shape}

MOSFET response was studied for the following regular field sizes: symmetric shapes of $80 \times 80,30 \times 30,24 \times 24,18 \times 18 \mathrm{~mm}^{2}$ and one asymmetric shape of $24 \times 36 \mathrm{~mm}^{2}$. A dose of $1 \mathrm{~Gy}$ was prescribed at the isocenter at a depth of $10 \mathrm{~cm}$ from the surface of the head phantom at $0^{\circ}$ (beam gantry incidence). Different gantry incidences were applied to provide different SSD with a multiplestatic converging beam similar to that used in radiosurgery. For all field sizes and gantry angles of $0,30,60,90,120$ and $240^{\circ}$, SSD were respectively: 90, 91.39, 93.03, 93.42, 92.66 and $90.41 \mathrm{~cm}$. For each field size-SSD combination, two doses values were determined by applying either $\mathrm{SC}_{\mathrm{m}}$ or $\mathrm{SC}_{\mathrm{c}}$ to the MOSFET reading. The dose results were compared using: standard deviation, mean value, deviation from prescribed dose. A coefficient of variation ( $\mathrm{CoV}=$ standard deviation/mean) was calculated to study distribution of measurements for each field size studied.

Table 1

MOSFET lifetime intervals considered for reproducibility study of a batch.

\begin{tabular}{|c|c|c|c|c|c|}
\hline$k$ th MOSFET & 1 & 2 & 3 & 4 & 5 \\
\hline $\begin{array}{l}\text { Ages intervals } \\
{[\mathrm{mV}]}\end{array}$ & $\begin{array}{l}{[0 ; 1820] \text { and }} \\
{[18,200 ; 20,000]}\end{array}$ & $\begin{array}{l}{[3640-5460] \text { and }} \\
{[16,380 ; 18,200]}\end{array}$ & $\begin{array}{l}5460 ; 7280] \text { and } \\
{[14,560 ; 16,380]}\end{array}$ & $\begin{array}{l}{[7280 ; 9100] \text { and }} \\
{[12,740 ; 14,560]}\end{array}$ & $\begin{array}{l}{[9100 ; 10,920] \text { and }} \\
{[10,920 ; 12,740]}\end{array}$ \\
\hline
\end{tabular}


Irregular shape

As shown in Fig. 2, four different target volumes were delineated and located at different depths from the surface of the head phantom.

The prescribed isocenter dose was $1 \mathrm{~Gy}$. As previously seen, different SSD were obtained by varying gantry angles. On a geometrical plane $\mathrm{P}$, passing through the isocenter and orthogonal to the beam axis for a given gantry angle, the field size is defined by the MLC with target 2D boundaries of $1 \mathrm{~mm}$ margin. From each irregular shape defined with MLC, the equivalent square field, $A$, was calculated by the Sterling-Worthley formula [21,22].

For each beam studied, the corresponding SSD was extracted from TPS. These last parameters were used as entrance parameters to find appropriate $\mathrm{SC}_{\mathrm{m}}$ and $\mathrm{SC}_{\mathrm{c}}$ to apply to MOSFET reading. At a $0^{\circ}$ gantry angle, equivalent field sizes corresponding to the 4 delineated target volumes were: $32.7 \times 32.7,22.4 \times 22.4$ $19.7 \times 19.7$ and $18.2 \times 18.2 \mathrm{~mm}^{2}$.

- For the two first PTV, beam gantry angles were $0,10,30,60,90$, 120 and $240^{\circ}$, and the corresponding SSD were: $90.02,90.08$, $91.39,93.03,93.42,92.66$ and $90.41 \mathrm{~cm}$ respectively.

- For the 3rd, beam gantry angles were $0,10,30,60,90,120$ and $240^{\circ}$, and the corresponding SSD were: 93.02, 93.05, 93.31, 94.2, 93.86, 92.22 and $90.11 \mathrm{~cm}$ respectively.

- For the 4th, beam gantry angles were $0,30,90,120,263,320$ and $348^{\circ}$, and the corresponding SSD were: $97.02,96.76,95.48$, 93.03, $93.29,96.31$ and $96.98 \mathrm{~cm}$ respectively.

The results were obtained and compared using same methods as described above.

\section{Results}

\section{Calibration}

In the study of the influence of build-up cap dimensions on MOSFET response, for field sizes from $98 \times 98$ to $18 \times 18 \mathrm{~mm}^{2}$ the progression of the overall charge particle equilibrium process ranged from 38.1 to $42.7 \%$ respectively at a depth of $7.1 \mathrm{~mm}$. The amount of the percentage depth dose released to the detector ranged from $92.1 \%$ to $94.3 \%$. Dose attenuation due to the sensor equipped with build-up cap was $2.10 \% \pm 0.05 \%$ for all field sizes. In the study of MOSFET recovery time, maximal variability of $\left(\mathrm{SC}_{\mathrm{m}}\right)_{\mathrm{SSD}}$, A was $2.4 \%$ for a calibration inter-measurement time lapse of $3 \mathrm{~min}$ against $1.8 \%$ for $5 \mathrm{~min}$ for all square field sizes at $\mathrm{SSD}=98.5 \mathrm{~cm}$. Table 2 reports the mean of Measured Sensitivity Coefficient $\left(\mathrm{SC}_{\mathrm{m}}\right)$ and associated variability obtained at $\mathrm{SSD}=98.5 \mathrm{~cm}$ on the range of field size studied for inter-measurement interval of 3 and $5 \mathrm{~min}$. Batch uniformity was found to be within $2.1 \%$. With complete calibration, 108 measurements have to be performed to explore all field size and SSD combinations. The corresponding calibration time was about $9 \mathrm{~h}$ for a long waiting time of 5 min against $7 \mathrm{~h}$ for a short waiting time of $3 \mathrm{~min}$. With optimized calibration, the number of measurements required can be significantly reduced down to 18 , since SCs are acquired only at SSD $=98.5 \mathrm{~cm}$ for all field sizes. The SC linking other field sizes and SSD combinations were calculated with the use of inverse square law. Thus, the optimized calibration time was only about $1 \mathrm{~h} 32 \mathrm{~min}$ for a long waiting time of $5 \mathrm{~min}$. Thus, the actual calibration time was only about $1 \mathrm{~h}$ $32 \mathrm{~min}$ for a long waiting time of $5 \mathrm{~min}$.

Figure 3 shows matrices $M_{m}$ (a) and $M_{c}$ (b) from 2D interpolation.

Highest variations were encountered for:

- $18 \times 18$ to $42 \times 42 \mathrm{~mm}^{2}$ field sizes, SSD $=97 \mathrm{~cm}$, range [-1\%; $-3.5 \%]$

- $18 \times 18$ to $98 \times 98 \mathrm{~mm}^{2}$ field sizes, SSD $=95 \mathrm{~cm}$, range $[0 \% ;+3.23 \%]$.

Table 3 reports the deviation between calculated shift values with the inverse square law and mean of 3 MOSFET measurements. For a given SSD or square field size, mean deviation was found to be $|1.55 \%|$ for all combinations. All deviations ranged from $-3.50 \%$ to $+3.23 \%$. A strong correlation was observed between measured and calculated shift values $\left(R^{2}=0.983\right)$ for all field sizes.

\section{Clinical validation measurements}

\section{Regular shape}

For clinical measurements for both regular and irregular field shapes performed on the head phantom, the total in-vivo measurement time was 3 h 24 min. Figure 4 plots the measured dose normalized to the prescribed dose [\%] for regular fields at different gantry angulations when $\mathrm{SC}_{\mathrm{c}}$ were applied to the MOSFET reading. Using $\mathrm{SC}_{\mathrm{m}}$, the mean deviation is $|1.70 \%|$ for all SSD and the whole range of regular field sizes explored. All measurements are spread between $-2.03 \%$ and $+5.24 \%$ of the prescribed dose. The CoV is equal to $1.77 \%$. Using $\mathrm{SC}_{\mathrm{c}}$ the mean deviation was $|1.71 \%|$. All calculated doses are spread between $-2.60 \%$ and $+5.24 \%$. CoV is equal to $1.82 \%$.

\section{Irregular shape}

Figure 5 shows the results for the measured dose normalized to the prescribed dose [\%] for irregular fields at different gantry angulations when $\mathrm{SC}_{\mathrm{c}}$ were applied to the MOSFET reading. Using $\mathrm{SC}_{\mathrm{m}}$, the mean deviation was $|2.28 \%|$ for all SSD and the whole range of irregular field sizes explored. All measurements are spread between $-7.62 \%$ and $+1.3 \%$. The $\mathrm{CoV}$ is equal to $2.08 \%$. Using $\mathrm{SC}_{\mathrm{c}}$ the mean deviation was $|2.53 \%|$ for all SSD and the whole range of irregular field sizes explored. All calculated doses are spread out

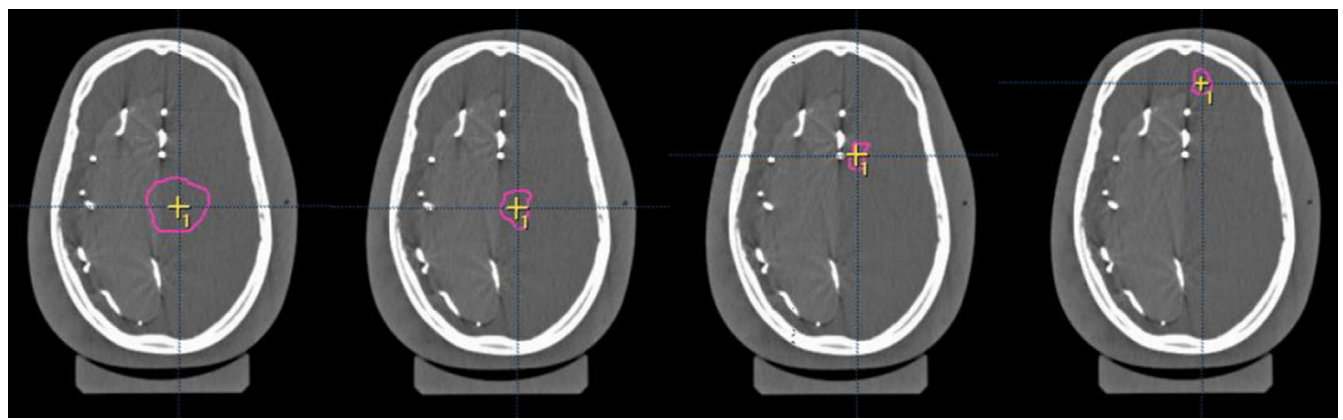

Figure 2. From left to right, transversal slices with the four different target volumes delineated on TPS 
Table 2

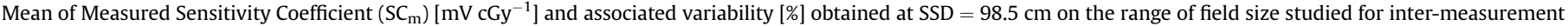
interval of 3 and $5 \mathrm{~min}$.

\begin{tabular}{|c|c|c|c|c|c|c|c|c|c|c|c|c|}
\hline & \multicolumn{12}{|c|}{ Square field size $\left(\mathrm{mm}^{2}\right)$} \\
\hline & \multicolumn{2}{|c|}{$18 \times 18$} & \multicolumn{2}{|c|}{$24 \times 24$} & \multicolumn{2}{|c|}{$30 \times 30$} & \multicolumn{2}{|c|}{$42 \times 42$} & \multicolumn{2}{|c|}{$80 \times 80$} & \multicolumn{2}{|c|}{$98 \times 98$} \\
\hline & $\mathrm{SC}_{\mathrm{m}}$ & Var & $\mathrm{SC}_{\mathrm{m}}$ & Var & $\mathrm{SC}_{\mathrm{m}}$ & Var & $\mathrm{SC}_{\mathrm{m}}$ & Var & $\mathrm{SC}_{\mathrm{m}}$ & Var & $\mathrm{SC}_{\mathrm{m}}$ & Var \\
\hline $3 \mathrm{~min}$ & 0.96 & 2.30 & 0.97 & 1.37 & 0.94 & 2.36 & 0.93 & 1.29 & 0.92 & 0.96 & 0.93 & 1.38 \\
\hline $5 \mathrm{~min}$ & 0.97 & 0.59 & 0.98 & 1.80 & 0.95 & 0.61 & 0.95 & 1.64 & 0.93 & 1.06 & 0.92 & 1.65 \\
\hline
\end{tabular}

between $-7.0 \%$ and $+1.49 \%$. $\mathrm{CoV}$ is equal to $1.82 \%$. For all field sizes (except one), $\mathrm{CoV}$ was smaller using $\mathrm{SC}_{\mathrm{c}}[-7.00 \% ;+1.49 \%]$, against [-7.62\%; $+1.3 \%]$ using $\mathrm{SC}_{\mathrm{m}}$. Results obtained using either $\mathrm{SC}_{\mathrm{m}}$ or $\mathrm{SC}_{\mathrm{c}}$ are summarized in Table 4.

\section{Discussion}

We proposed and optimized a method to calibrate MOSFET located at the surface, applicable to shaped-beam radiosurgery for field sizes of $98 \times 98$ to $18 \times 18 \mathrm{~mm}^{2}$.

The influence of the RW3 build-up cap on the MOSFET response was considered. The results showed that the amplitude of the equivalent dose deposition (\%) released to the MOSFET is about $2 \%$ over the range of field sizes studied since the depth of maximum dose shifts toward the surface when field sizes diminishes [18]. From $98 \times 98$ to $18 \times 18 \mathrm{~mm}^{2}$ field sizes, MOSFET response should be corrected by the variation of the equivalent dose deposition released to the detector. An advantage of our optimized calibration method is that this additional correction factor is built into the SC values obtained in the several field sizes explored at the calibration step. On average and irrespective of field size, the dose attenuation due to the detector equipped with the RW3 build-up cap was near $2 \%$.

This attenuation value is small compared to in-vivo dosimetry studies conducted with diodes or with OSLDs [23-25]. It can also be compared to the $6 \%$ reported by Morton et al. using MOSFET covered with an aluminum build-up cap [14].

In conventional radiotherapy in-vivo measurements are performed for each beam. However, as the dose actually received by the patient is attenuated by the presence of the dosimeter, measurements are not performed on each fraction in conventional radiotherapy. Indeed, according to the guidelines published in AAPM report No 87 , a global dose attenuation of $0.5 \%$ remains negligible and is equivalent to a "cold spot" of $10 \%$ obtained using a diode as in-vivo dosimeter in one fraction out of a total of 30 [26].

However, in stereotactic radiosurgery, most treatments are delivered in a single dose. Therefore, to minimize the shadowing effect, and reach clinical acceptability, we suggest for example:

- To measure a single beam (the first one, and if possible the least dose contributive).

- To measure all the beams delivered but only for a fraction of their duration.

Over the whole range of field sizes studied, the characteristics of the build-up cap were chosen to minimize variations of progression of the overall process of charge particle equilibrium, variations of dose deposition along the beam axis, dose attenuation and to satisfy geometrical conditions of the field at the phantom surface. Therefore, we assume that performing entrance in-vivo dosimetry in small fields, the build-up cap dimensions have to ensure only partial charge particle equilibrium.

A post-irradiation time of $30 \mathrm{~s}$ was chosen to perform MOSFET reading but, a $10 \mathrm{~s}$ post-irradiation time was reported [27]. However, we assume that $10 \mathrm{~s}$ are not a sufficient delay to perform detector reading since a signal drift occurs in the first seconds following the end of irradiation. This effect was identified via the creep up effect, as the drift between two post-irradiations readings - one taken immediately after irradiation, and a second after a time delay. In fact as it at $30 \mathrm{~s}$ the signal drift due to charge movement in the detector is only about $1 \mathrm{mV}$ which is in the order of the sensitivity bias chosen (e.g. $1 \mathrm{mV} \mathrm{cGy}^{-1}$ ) for this study [11]. We studied consistent time intervals ( 3 and $5 \mathrm{~min}$ ) between consecutive irradiations to minimize dose reading variability due to fading effect. It is known that the radiation stored in a MOSFET fades over time $[27,28]$. In our case, the most accurate $\mathrm{SC}_{\mathrm{m}}$ values were
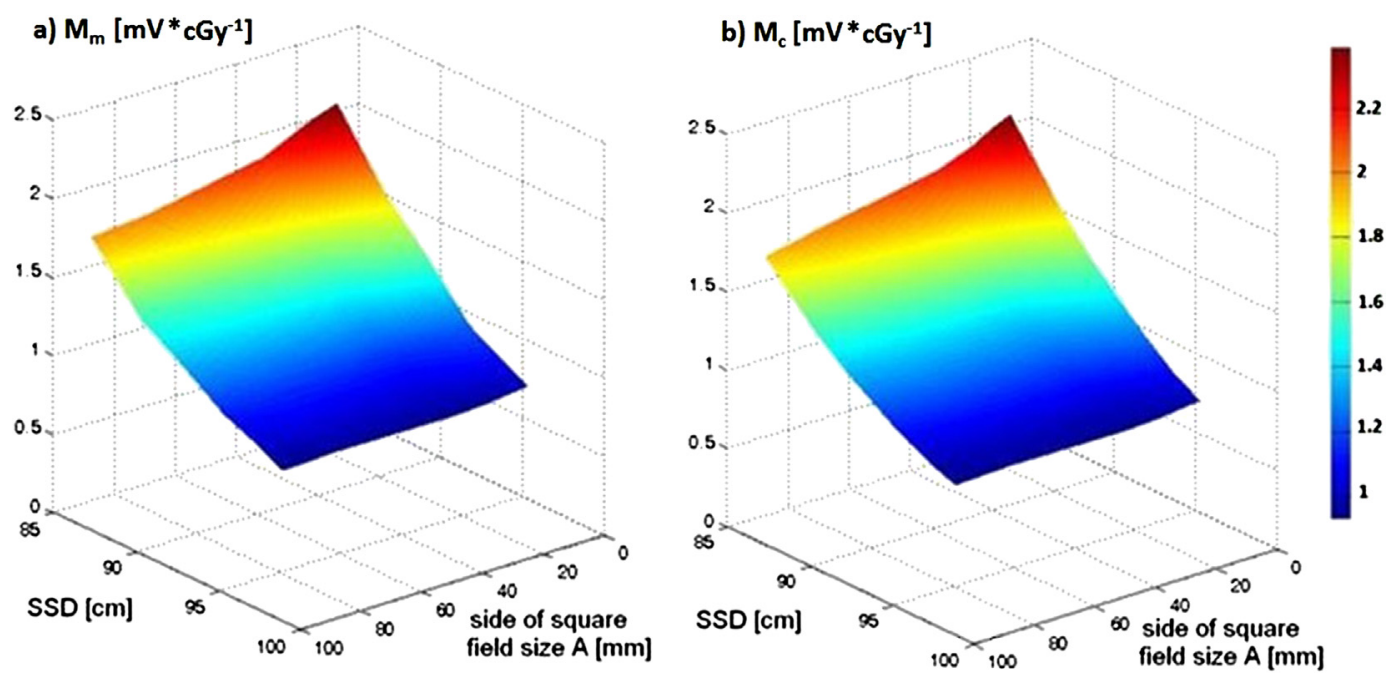

Figure 3. $M_{\mathrm{m}}$ (a) and $M_{\mathrm{c}}$ (b) obtained from 2D interpolation between SSD and field size. 
Table 3

Deviation [\%] between voltage shifts calculated with the inverse square law and mean measurements for a given SSD-field size combination.

\begin{tabular}{lcccccc}
\hline SSD $[\mathrm{cm}]$ & \multicolumn{5}{l}{ Square field size $\left[\mathrm{mm}^{2}\right]$} \\
\cline { 2 - 7 } & $18 \times 18$ & $24 \times 24$ & $30 \times 30$ & $42 \times 42$ & $80 \times 80$ & $98 \times 98$ \\
\hline 87 & -0.79 & -0.34 & 0.81 & -0.21 & -0.38 & 1.97 \\
90 & -1.07 & -1.47 & -0.20 & -0.96 & -1.76 & -1.10 \\
92 & 0.94 & 0.16 & 1.82 & 2.19 & 0.93 & 1.80 \\
95 & -3.50 & -3.21 & -3.16 & -2.13 & 0.45 & 1.15 \\
97 & 0.99 & 2.03 & 3.10 & 1.48 & 3.04 & 3.23 \\
98.5 & 0.00 & 0.00 & 0.00 & 0.00 & 0.00 & 0.00 \\
\hline
\end{tabular}

obtained for a 5 -min delay (using a 5 min delay, maximal variability was $1.8 \%$ against $2.40 \%$ using a 3 min delay). This could be explained by the low holes mobility that increases their probability of all being trapped and restabilized with time. Moreover, frequent calibration of the device is usually recommended since SC values decrease with high threshold voltage due to previous irradiation. This effect is especially important in low energy beams but quite stable for high energy irradiations $(E \geq 1 \mathrm{MeV})$ because of an alteration of the effective electric field applied to the MOSFET [29]. Prior to this work, we first studied the influence of accumulated dose on MOSFET sensitivity. We observed no variation in sensitivity with accumulated dose during MOSFET lifetime. This result (not shown) is similar to those obtained by Jornet et al. [30].

Batch uniformity $(2.1 \% \pm 0.05 \%)$ was found to be similar to the range provided by the manufacturer (2\%) [16] and also to results of other studies [31].

In this work, for the method exploring all field size-SSD combinations, the total calibration time was between 7 and $9 \mathrm{~h}$. In the literature, the time constraint for detector calibration is often mentioned as a crucial element to be taken into account when invivo dosimetry is employed for treatment verification in a clinical activity center. However, comparison to other methodologies is difficult due to lack of papers detailing total detector calibration time. Thus, it has been mentioned that EPID dosimetry is timeconsuming and complicated due to the assessment of absolute dose from in-vivo measurement in radiotherapy [32]. The use of TLD was reported to be more time-consuming, since it requires complicated annealing, with careful hand calibration of the processor [33]. For diodes, largely described as in-vivo dosimeters, calibration seems to be faster. However, the introduction of the diode response factors, to calculate the actual dose measured can be a time-consuming

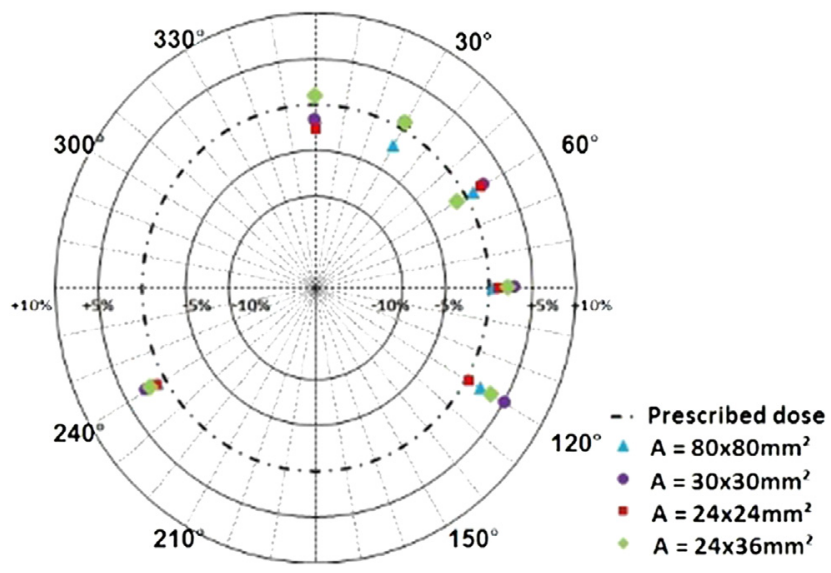

Figure 4. Measured dose normalized to the prescribed dose for regular shapes using $\mathrm{SC}_{\mathrm{c}}$. Dotted lines represent prescribed dose whereas the colored symbols show the data measured for each series of field size explored.

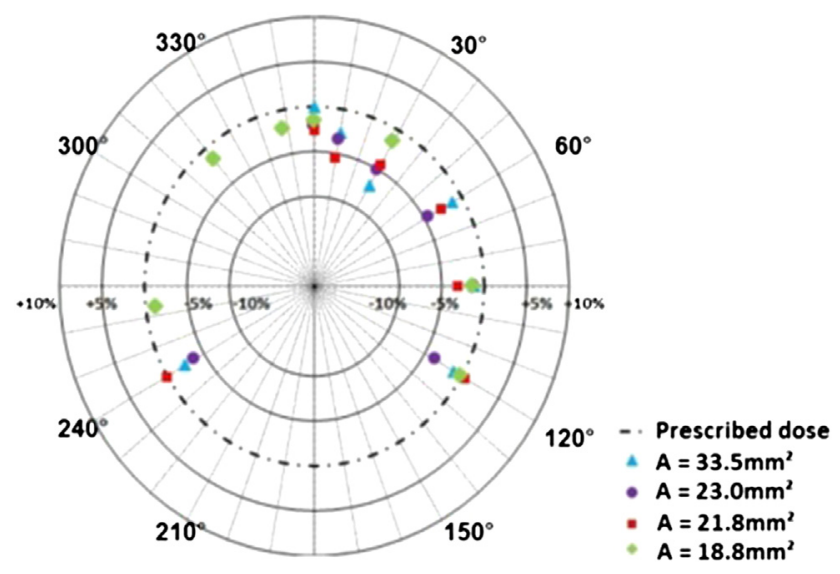

Figure 5. Measured dose normalized to the prescribed dose for irregular fields [mm] using $\mathrm{SC}_{\mathrm{c}}$. Dotted lines represent prescribed dose and the colored symbols show the data measured for each field size series $A$ explored.

process [34]. In our work, reducing the waiting time from 5 to $3 \mathrm{~min}$ decreases total calibration time by 2 h for complete MOSFET calibration. Moreover, a shorter waiting time of $3 \mathrm{~min}$ induced maximal variability on $\mathrm{SC}_{\mathrm{m}}$ accuracy (2.4\% against $1.8 \%$ ). With our optimized calibration method, this time difference would only be of the order of $17 \min$ ( $1 \mathrm{~h} 32$ for 5 min waiting time against $1 \mathrm{~h} 15$ for 3 min, results not shown), since the total calibration time and number of measurements to perform are divided by six. Therefore, applying an inter-measurement cycle interval of $5 \mathrm{~min}$ for optimized MOSFET calibration remains reasonable for us in a clinical activity, with regard to the SC accuracy improvement obtained. Therefore, calibrating only one MOSFET from a given batch from $98 \times 98$ down to $18 \times 18 \mathrm{~mm}^{2}$, just once with an intermeasurement time lapse of 5 min seems to be the best compromise between $\mathrm{SC}_{\mathrm{m}}$ determination accuracy and calibration time for use in stereotactic radiosurgery. We first intended to validate a calibration method with a well-known MOSFET device (with buildup cap) known to be efficient for in-vivo surface measurements in IMRT $[13,31,35]$, where small fields are encountered. We are also currently working on microMOSFETs. This work on MOSFETs was necessary because, to our knowledge there is no published data investigating small field sizes with standard MOSFETs in stereotactic radiosurgery.

Indeed, a commercial RW3 build-up cap exists for the use of the MOSFETs as entrance detectors. In this study, we assumed that the minimal square field size efficiently explored has to be greater than the build-up cap dimensions (diameter of $13 \mathrm{~mm}$ ) [2]. Therefore, the cap size constitutes the limit for the smallest filed sizes used in radiosurgery. The smallest field size explored in this study was $18 \times 18 \mathrm{~mm}^{2}$ as defined at the isocenter plane. At the surface, the side of the equivalent field size at the phantom surface ranges from 17.64 to $15.66 \mathrm{~mm}$, i.e. remains larger than the build-up cap

Table 4

Results obtained using either $\mathrm{SC}_{\mathrm{m}}$ or $\mathrm{SC}_{\mathrm{c}}$ for each square field size series $\mathrm{A}$ explored in irregular shapes.

\begin{tabular}{|c|c|c|c|c|c|c|c|c|}
\hline & \multicolumn{8}{|c|}{ Average of each equivalent field size series $\left[\mathrm{mm}^{2}\right]$} \\
\hline & \multicolumn{2}{|c|}{$33.5 \times 33.5$} & \multicolumn{2}{|c|}{$23.0 \times 23.0$} & \multicolumn{2}{|c|}{$21.8 \times 21.8$} & \multicolumn{2}{|c|}{$18.8 \times 18.8$} \\
\hline & $\mathrm{SC}_{\mathrm{m}}$ & $\mathrm{SC}_{\mathrm{c}}$ & $\mathrm{SC}_{\mathrm{m}}$ & $\mathrm{SC}_{\mathrm{c}}$ & $\mathrm{SC}_{\mathrm{m}}$ & $\mathrm{SC}_{\mathrm{c}}$ & $\mathrm{SC}_{\mathrm{m}}$ & $\mathrm{SC}_{\mathrm{c}}$ \\
\hline Mean [cGy] & 97.67 & 97.77 & 97.11 & 96.16 & 98.36 & 97.45 & 98.61 & 98.66 \\
\hline Std deviation [cGy] & 2.56 & 2.28 & 1.50 & 0.92 & 2.10 & 2.19 & 1.71 & 0.61 \\
\hline $\mathrm{CoV}[\%]$ & 2.62 & 2.34 & 1.54 & 0.96 & 2.13 & 2.25 & 1.73 & 0.61 \\
\hline Mean deviation [\%] & 2.50 & 2.23 & 2.89 & 3.84 & 2.01 & 2.73 & 1.74 & 1.34 \\
\hline
\end{tabular}


diameter, for SSDs ranging from $98.5 \mathrm{~cm}$ to $87 \mathrm{~cm}$. If smaller field sizes were explored, for example $12 \times 12 \mathrm{~mm}^{2}$ with the same SSDs, the side of the equivalent field size at the phantom surface would have been from 11.76 to $10.44 \mathrm{~mm}$, and could have led to larger beam disturbances using MOSFET TN-502RD with the commercial RW3 build-up cap.

Similarly, microMOSFET detectors, smaller than MOSFET, are efficient in-vivo dosimeters, but mostly reported for intracavity and skin dose measurements [36]. Hence, there is no commercial buildup cap available to perform in-vivo dosimetry with microMOSFET placed at the surface. Also, by placing a microMOSFET in the groove of a MOSFET build-up cap leads to an inacceptable air gap. Therefore, for microMOSFET, the build-up cap has to be home-made if surface measurements are to be envisaged.

The objective of the present work was first to develop and evaluate a calibration method suited to MOSFET technology and radiosurgery constraints using a robust device that had proved to be a valid option for surface measurements in small fields. MOSFET dosimeters have been demonstrated to be more suitable than the reference pinpoint chamber with vertical direction for dose measurement of small field sizes [23]. To minimize discrepancies between calibration and measurement setups, MOSFETs were calibrated at the surface since they should be positioned at the skin surface in clinical measurements.

An additional way to reduce the number of calibration measurements was to use the inverse square law to predict voltage shifts from each field size measurement obtained at SSD 98.5 , including small field conditions, since a good agreement between $\mathrm{SC}_{\mathrm{m}}$ and $\mathrm{SC}_{\mathrm{c}}$ values was observed and a strong correlation between measured and calculated voltage shifts $\left(R^{2}=0.983\right)$ was obtained. This SSD value was chosen because it is representative of usual calibration SSD encountered in in-vivo dosimetry at $6 \mathrm{MV}$, and represents the minimal depth of radiosurgery treatments. $\mathrm{SC}_{\mathrm{c}}$ were calculated for each field size-SSD combination for which $\mathrm{SC}_{\mathrm{m}}$ were measured i.e. from $98 \times 98$ to $18 \times 18 \mathrm{~mm}^{2}$ and 98.5 to $87 \mathrm{~cm}$. We consider the use of both inverse square law and SC measurements at all field sizes between $\left(98 \times 98\right.$ and $\left.18 \times 18 \mathrm{~mm}^{2}\right)$ as the best compromise to minimize number of measurements to be performed at the MOSFETs calibration step. Indeed, for typical field sizes encountered in stereotactic radiosurgery, it is difficult to accurately predict by a model the lack of scattered components that occurs for field sizes under lateral electronic disequilibrium conditions $[37,38]$. Indeed, with regard to microbeam metrology, high variability in Total Scatter Factors value is described in the small fields depending on the type or position of the reference dosimeter.

Moreover this statement is in agreement with a recent formalism for reference dosimetry of small and non-standard fields to avoid calibration to a unique field size but instead introduce intermediate field sizes [39]. To test our calibration method, different irradiation geometric conditions were applied on a realistic head phantom. Considering the time constraints of a clinical activity, processing time for in-vivo dosimetry measurements should be kept minimal so as not to overextend total treatment time. In this work, phantom study duration was estimated to be $3 \mathrm{~h}$ $24 \mathrm{~min}$. We determined the shortest waiting time ensuring fast measurement sequences with a shaped beam. With intracranial radiosurgery shaped-beam treatment, it only takes 3 min to set up beam parameters before delivery and perform MOSFET positioning. Therefore, no added waiting time was needed to perform MOSFET irradiation. In a real clinical situation, dose reading would be obtained $30 \mathrm{~s}$ after delivery. In consequence, the total processing time to perform in-vivo measurements per treatment would essentially be conditioned by beam setup time. The method described in this paper, i.e. entrance dose measurement on the beam axis at the skin surface of the patient for stereotactic radiosurgery, is difficult to apply to the Cyberknife ${ }^{\circledR}$, since the treatment times are already long and may require about 300 static non-isocentric beams for typical intracranial cases. It is therefore not feasible to wait between each beam for the MOSFET to stabilize. Indeed, using the Cyberknife $^{\circledR}$, the treatment time for single-shot stereotactic radiosurgery is 3-4 h and ranges between 60 and 90 min per session for fractionated treatment. However, the use of MOSFET for in-vivo dosimetry has been reported for Cyberknife application with detectors implanted in the tumour [40]. In regular fields, SSD interpolation led to good results since mean deviation and $\mathrm{CoV}$ were $<2 \%$. Equivalent results were found using $\mathrm{SC}_{\mathrm{c}}$.

In fact angular dependence exists for MOSFETs, but here it is not a concern because the relative angle between the detector and the beam axis was always $0^{\circ}$. Anisotropy of in-vivo dosimeters is well known and is mainly explicable for MOSFET by beam attenuation due to detector geometry. Several studies published before 1999 reported strong anisotropy when TN-502RD detectors are used. Indeed, variations of up to $17 \%$ (on $360^{\circ}$ angles), $28 \%$ for beam angles between 0 and $180^{\circ}$ and $7 \%$ for beam angles between 0 and $90^{\circ}$ have been reported [41-44]. However, angular dependency was only $3-3.5 \%$ for the most recent isotropic models [31,40,45] in electronic equilibrium conditions. For irregular field shapes, 2D interpolation between field size and SSD to find appropriate sensitivity coefficients using the inverse square law and the Sterling-Worthley formula gave satisfactory results for in-vivo dosimetry ( $<5 \%$ ). Using $\mathrm{SC}_{\mathrm{m}}$, the mean deviation was $|2.28 \%|$ versus $|2.53 \%|$ using $\mathrm{SC}_{\mathrm{c}}$. Unexpectedly, the largest mean deviation using $\mathrm{SC}_{\mathrm{c}}$ was $3.84 \%$ and was encountered not for the smallest but for intermediate field sizes $\left(23 \times 23 \mathrm{~mm}^{2}\right)$. However, the CoV value remained lower using $\mathrm{SC}_{\mathrm{c}}$ for this field size series. This seemed to show that an underestimation of the delivered dose was introduced by the inverse square law itself. For field sizes below $30 \times 30 \mathrm{~mm}^{2}$, it has been described that loss of lateral electronic equilibrium is not taken into account when SSD decreases [1]. This phenomenon had a greater effect when the edges of the field approached lateral electronic equilibrium conditions (field sizes ranging from $20 \times 20 \mathrm{~mm}^{2}$ to $30 \times 30 \mathrm{~mm}^{2}$ ), and could then explain differences between calculated doses obtained using $\mathrm{SC}_{\mathrm{m}}$ and $\mathrm{SC}_{\mathrm{c}}$. For irregular shapes, underestimation of the overall dose was observed using either $\mathrm{SC}_{\mathrm{m}}$ or $\mathrm{SC}_{\mathrm{c}}$. This effect could be mainly caused by the use of the equivalent square Sterling-Worthley formula. Thus, this was not the case when the equivalent square calculation was not required (regular fields) and when the lateral electronic equilibrium was reached at least in one dimension (case of the asymmetric field). Indeed, it has been reported that this formula failed to predict collimator exchange effects and losses of lateral electronic equilibrium in small fields [46]. Finally, our results seem to be acceptable for in-vivo dosimetry studies (deviation in irregular fields $<5 \%$ ) since they are similar to those obtained for IMRT plan verification for MOSFETs located at the surface with a build-up cap [13] and for phantom inserts [31].

\section{Conclusions}

With the inverse square law, it is possible to reduce the number of measurements required for MOSFET calibration for multiple typical field size and SSD combinations encountered in stereotactic radiosurgery. We have developed an optimized calibration method to find the best compromise between $\mathrm{SC}_{\mathrm{m}}$ determination accuracy and calibration time. Only one MOSFET from a given batch was calibrated just once with an inter-measurement time lapse of $5 \mathrm{~min}$ at a $\mathrm{SSD}=98.5 \mathrm{~cm}$ for several field sizes ranging from $98 \times 98 \mathrm{~mm}^{2}$ down to $18 \times 18 \mathrm{~mm}^{2}$. This optimized method preserves the useful life of the detector for in-vivo measurements in comparison to complete calibration. Good results were observed in shaped-beam 
radiosurgery for equivalent square field sizes of $18.8 \times 18.8 \mathrm{~mm}^{2}$ to $33.5 \times 33.5 \mathrm{~mm}^{2}$ on a realistic head phantom. Our results have shown that MOSFETs are suitable for use as dosimeters when located at the surface for irradiation fields of $98 \times 98 \mathrm{~mm}^{2}$ down to $18 \times 18 \mathrm{~mm}^{2}$ and SSD from 87 to $98.5 \mathrm{~cm}$. Moreover, this calibration method is also well suited for the multiple-static converging beam technique. Nevertheless, further geometric conditions of irradiation should be explored to investigate a broader range of radiosurgery treatments and specifically, field sizes below $18 \times 18 \mathrm{~mm}^{2}$. One of the major perspectives of the present work could be investigations into the use of microMOFETS.

\section{Conflict of interest}

There is no conflict of interest related to this project.

\section{Acknowledgements}

The authors would like to acknowledge John Thomas and Abdelbasset Hallil from Best Medical Canada and Patrice Gatinois from Arplay Medical for their support on the technology of the mobileMOSFET system and for valuable discussions about the project.

\section{References}

[1] Duggan DM, Coffey 2nd CW. Small photon field dosimetry for stereotactic radiosurgery. Med Dosim 1998;23:153-9.

[2] Rice RK, Hansen JL, Svensson GK, Siddon RL. Measurements of dose distributions in small beams of 6-MV X-rays. Phys Med Biol 1987;32:1087-99.

[3] Bjarngard CF, Tsai JS, Rice RK. Doses on the central axes of narrow 6-MV X-ray beams. Med Phys 1990;17:794-9.

[4] Francescon P, Cora S, Cavedon C, Scalchi P, Reccanello S, Colombo F. Use of new types of radiochromic film, a new parallel plate chamber, MOSFETs and TLD 800 microcubes in the dosimetry of small beams. Med Phys 1998;25: 503-11.

[5] Serago CF, Houdek PV, Hartmann GH, Saini DS, Serago ME, Kaydee A. Tissue maximum ratios (and other parameters) of small circular 4, 6, 10, 15 and 24 MV X-ray beams for radiosurgery. Phys Med Biol 1992;37:1943-56.

[6] Kurjewicz L, Berndt A. Measurement of Gamma Knife ${ }^{\circledR}$ helmet factors using MOSFETs. Med Phys 2007;34:1007-12.

[7] Francescon P, Cora S, Cavedon C, Scalchi P. Application of a Monte Carlo based method for total scatter factors of small beams to new solid state micro-detectors. J Appl Clin Med Phys 2009;10:2939.

[8] Ramaseshan R, Kohlil KS, Zhang TJ, Lam T, Norlinger B, Hallil A, et al. Performance characteristics of a microMOSFET as in vivo dosimeter in radiation therapy. Phys Med Biol 2004;49:4031-48.

[9] Bloemen-van Gurp EJ, Minken AW, Mijnheer BJ, Dehing-Oberye CJ, Lambin P. Clinical implementation of MOSFET detectors for dosimetry in electron beams. Radiother Oncol 2006;80:288-95.

[10] Rosenfeld AB. MOSFET dosimetry on modern radiation oncology modalities. Radiat Prot Dosimetry 2002;101:393-8.

[11] Ramani R, Russell S, O’Brien P. Clinical dosimetry using MOSFETs. Int J Radiat Oncol Biol Phys 1997;37:959-64.

[12] Cherpak A, Studinski RC, Cygler JE. MOSFET detectors in quality assurance of tomotherapy. Radiother Oncol 2008;86:242-50.

[13] Varadhan R, Miller J, Garrity B, Weber M. In vivo prostate IMRT dosimetry with MOSFET detectors using brass buildup caps. J Appl Clin Med Phys 2006;7:22-32.

[14] Morton JP, Bhat M, Kovendy A, Williams T. Evaluation of MOSFETs for entrance dose dosimetry for 6 and $10 \mathrm{MV}$ photons with a custom made build up cap. Australas Phys Eng Sci Med 2007;30:120-6.

[15] Rosenfeld AB. Electronic dosimetry in radiation therapy. Radiat Meas 2007;41:S134-53.

[16] Scalchi P, Francescon P. Calibration of a MOSFET detection system for 6-MV in vivo dosimetry. Int J Radiat Oncol Biol Phys 1998;40:987-93.

[17] Thomson and Neilson Electronics Ltd. MOSFET calibration JIG, instructions for use. Ottawa, Canada: Thomson and Neilson Electronics Ltd.; 1996. TN \#102046.01.

[18] Podgorsak EB, Podgorsak MB. Quality assurance of external beam radiotherapy. In: Podgorsak EB, editor. Radiation oncology physics: a handbook for teachers and students. Vienna: International Atomic Energy Agency; 2003. p. 407-50.
[19] Morton JP, Bhat M, Williams T, Kovendy A. Clinical results of entrance dose invivo dosimetry for high energy photon in external beam radiotherapy using MOSFETs. Australas Phys Eng Sci Med 2007;30:252-9.

[20] Zhu XR. Entrance dose measurements for in-vivo diode dosimetry: comparison of correction factors for two types of commercial silicon diode detectors. J Appl Clin Med Phys 2000;3(1):101-7.

[21] Sterling TD, Perry H, Katz L. Automation of radiation treatment planning. IV. Derivation of a mathematical expression for the per cent depth dose surface of cobalt 60 beams and visualization of multiple field dose distributions. $\mathrm{Br} \mathrm{J}$ Radiol 1964;37:544-50.

[22] Worthley B. Equivalent squares of rectangular fields. Br J Radiol 1966;39:559.

[23] Kohno R, Hirano E, Nishio T, Miyagishi T, Goka T, Kawashima M, et al. Dosimetric evaluation of a MOSFET detector for clinical application in photon therapy (1). Radiol Phys Technol 2008:55-61.

[24] Kohno R, Nishio T, Miyagishi T, Hirano E, Hotta K, Kawashima M, et al. Experimental evaluation of a MOSFET dosimeter for proton dose measurements. Phys Med Biol 2006;51:6077-86.

[25] Lavallée MC, Gingras L, Beaulieu L. Energy and integrated dose dependence of MOSFET dosimeter sensitivity for irradiation energies between $30 \mathrm{kV}$ and ${ }^{60}$ Co. Med Phys 2006;33:3683-9.

[26] AAPM report no. 87, 2005, Diode in-vivo dosimetry for patients receiving external beam radiation therapy, AAPM. Report of Task Group 62.

[27] Jursinic PA, Yahnke CJ. In vivo dosimetry with optically stimulated luminescent dosimeters, OSLDs, compared to diodes; the effects of buildup cap thickness and fabrication material. Med Phys 2011;38:5432-40.

[28] Sen A, Parsai EI, McNeeley SW, Ayyangar KM. Quantitative assessment of beam perturbations caused by silicon diodes used for in vivo dosimetry. Int J Radiat Oncol Biol Phys 1996;36:205-11.

[29] Colussi VC, Beddar AS, Kinsella TJ, Sibata CH. In vivo dosimetry using a single diode for megavoltage photon beam radiotherapy: implementation and response characterization. J Appl Clin Med Phys 2001;2:210-8.

[30] Jornet N, Carrasco P, Jurado D, Ruiz A, Eudaldo T, Ribas M. Comparison study of MOSFET detectors and diodes for entrance in vivo dosimetry in $18 \mathrm{MV} \mathrm{X}$-ray beams. Med Phys 2004;31(9):2534-42.

[31] Chuang CF, Verhey LJ, Xiaa P. Investigation of the use of MOSFET for clinical IMRT dosimetric verification. Med Phys 2002;29:1109-15.

[32] Higgins PD, Alaei P, Gerbi BJ, Dusenbery KE. In vivo diode dosimetry for routine quality assurance in IMRT. Med Phys 2003;30(12):3118-23.

[33] Kinhikar RA, Sharma PK, Tambe CM, Mahantshetty UM, Sarin R, Deshpande DD, et al. Clinical application of a one dose MOSFET for skin dose measurements during internal mammary chain irradiation with high dose rate brachytherapy in carcinoma of the breast. Phys Med Biol 2006;51(14): 263-8.

[34] Alecu R, Alecu M, Ochran TG. A method to improve the effectiveness of diode in vivo dosimetry. Med Phys 1998;25(5):746-9.

[35] Marcié S, Charpiot E, Bensadoun RJ, Ciais G, Herault J, Costa A, et al. In vivo measurements with MOSFET detectors in oropharynx and nasopharynx intensity modulated radiation therapy. Int J Radiat Oncol Biol Phys 2005;61: 1603-6.

[36] Cygler JE, Saoudi A, Perry G, Morash C, Cohan CE. Feasibility study of using MOSFET detectors for in vivo dosimetry during permanent low dose rate prostate implants. Radiother Oncol 2006;80:296-301.

[37] Das J, Downes MB, Kassaee A, Tochner Z. Choice of radiation detector in dosimetry of stereotactic radiosurgery-radiotherapy. J Radiosurg 2000;3: $177-85$.

[38] Martens C, De Wagter C, De Neve W. The value of the PinPoint ion chamber for characterization of small field segments used in intensity-modulated radiotherapy. Phys Med Biol 2000;45:2519-30.

[39] Alfonso R, Andreo P, Capote R, Huq MS, Kilby W, Kjäll P, et al. A new formalism for reference dosimetry of small and nonstandard fields. Med Phys Nov 2008;35(11):5179-86.

[40] Scalchi P, Righetto R, Cavedon C, Francescon P, Colombo F. Direct tumor in vivo dosimetry in highly-conformal radiotherapy: a feasibility study of implantable MOSFETs for hypofractionated extracranial treatments using the Cyberknife system. Med Phys 2010;37(4):1413-23.

[41] Bower MW, Hintenlang DE. The characterization of a commercial MOSFET dosimeter system for use in diagnostic X-ray. Health Phys 1998;75:197-204.

[42] Meiler RJ, Podgorsak MB. Characterisation of the response of commercial diode detectors used for in vivo dosimetry. Med Dosim 1997:22(1):31-7.

[43] Rikner G, Grussel E. Patient dose measurements in photon fields by means of silicon semiconductor detectors. Med Phys 1987;14(5):870-3.

[44] Rosenfeld AB, Carolan MG, Kaplan GI, Allen BJ, Khivrich VI. MOSFET dosimeters: the role of encapsulation on dosimetric characteristics in mixed gammaneutron and megavoltage X-ray fields. IEEE Trans Nucl Sci 1995;NS-42(6): 1870-7.

[45] Consorti R, Petrucci A, Fortunato F Soriani A, Marzi S, Jaccarino G, et al. In vivo dosimetry with MOSFETs: dosimetric characterization and first clinical results in intraoperative radiotherapy. Int J Radiat Oncol Biol Phys 2005;63(3):952-60.

[46] Thomas SJ, Eaton DJ, Tudor GSJ, Twyman NI. Equivalent squares for small field dosimetry. Br J Radiol 2008;81:897-901. 